\title{
Research on Reliability Assessment Based on the Multi-Source Information Fusion Technology with Improved Inheritance Factor
}

\author{
Jing-Bo GAla , Gao-Feng WEI \\ College of Aerospace and Civil Engineering, Harbin Engineering University, Harbin 150001, \\ Heilongjiang, China \\ agaijingbo@hrbeu.edu.cn
}

\begin{abstract}
Keywords: Reliability assessment, Information fusion technology, Inheritance factor, Complex system with small samples.
\end{abstract}

\begin{abstract}
In order to solve the problems of the classical system reliability assessment method in dealing with complex system with small samples, such as multilevel approximation, insufficiency and singleness of information, take the success or failure products as an example, the theory of multisource reliability information fusion based on inheritance factor is introduced to solve the problem of reliability assessment of complex system with small samples. The method of determining the inheritance factor is improved, and a method to determine the inheritance factor by the goodness of fit is given. Taking a small samples reliability assessment of a certain type of solid rocket motor as an example, the rationality of the present method and its application prospect are indicated.
\end{abstract}

\section{The Presentation of the Question}

In recent years, with the development of science and technology industry, a variety of large-scale complex equipment system is constantly put into practical use. For this kind of large-scale complex equipment system, it is often faced with a short development cycle, and it cannot be carried out a large number of system level reliability test due to human, material and financial constraints. Obviously, the problem that the system level test data of the large scale complex system is small samples makes the classical reliability assessment method based on mathematical statistics cannot get more realistic results. So, an approximative system reliability assessment method of pyramid structure is proposed. This method has been well applied in engineering, but the single source of information and the existence of multilevel approximation lead to the "accuracy" crisis. Especially for the test data of the unit and the system is all small samples, the approximative system reliability assessment method of pyramid structure is powerless.

In fact, there is a great deal of useful information for evaluating the reliability of the system in each link of the life profile of the product. To scientifically evaluate the reliability of the product, the potential of multi-source reliability information should be fully excavated. Meanwhile, the intersection, multi-source and varied population of the reliability information increase the difficulty of processing and utilization of reliability information. With such a strong demand driven, his research of information fusion technology has become the key to improve the utilization of reliability information, and to carry out scientific reliability assessment.

\section{The Theory Based on the Multi-Source Reliability Information Fusion Technology}

\section{The Reliability Information for Information Fusion}

In the fusion of reliability information, there are the following information: expertise information, components and subsystem information and reliability growth information, etc.

Expertise information fusion is a certain understanding of product reliability which is fused by the experience of a number of experts. Component and subsystem information fusion is to fuse the reliability test information of each component and subsystem into the system level reliability information. Reliability growth information fusion is to fuse multistage reliability growth information into overall reliability understanding by building dynamic growth model. 
The folding of these three kinds of information is usually based on information theory, and the specific methods are given in the literature [1] and [2].

\section{The Establishment of Prior Distribution Based on Inheritance Factor}

In reliability engineering, the reliability information is usually obtained through different ways or under different test conditions. This leads to the reliability information may not belong to the same population. It will introduce the subjective factors to the assessment results if the sources of information are considered to be from the same population, which will lead to the deviation of the assessment results. Especially in the system field test information is small samples, the impact is disastrous.

For the possible varied population of the multi-source reliability information, we take the success or failure products as an example to show how to solve it. Corresponding prior distribution is built according to the performance of different reliability information. Then, the concept of inheritance factor is introduced, and the reliability assessment method based on multi-source information fusion technology is discussed. This overcomes the shortcomings of the traditional Bayes method, which only takes into account the similarity and inheritance of the two samples but ignored the varied population.

In the application of reliability engineering, the conjugate distribution, Beta distribution, is usually used as the prior distribution of the product of the success or failure.Its probability density function is

$$
\pi_{i}(R)=\frac{\Gamma\left(a_{i}+b_{i}\right)}{\Gamma\left(a_{i}\right) \Gamma\left(b_{i}\right)} R^{a_{i}-1}(1-R)^{b_{i}-1}
$$

In the formula, $a_{i}, b_{i}$ are hyper-parameters of prior distribution. They are determined by the corresponding reliability information.

What needs to be explained is that the reliability information is expressed by $a_{i}, b_{i}$. If the reliability information is given in the form of success and failure times, that is $\left(s_{i}, f_{i}\right)$, at the same time if the reliability information passed the compatibility test, the hyper-parameters can be directly taken as

$$
\left(a_{i}, b_{i}\right)=\left(s_{i}, f_{i}\right)
$$

It is generally considered that the reliability information and the system field test information come from two different parent population. In order to reduce the influence of the of the varied population on the reliability assessment results, make the use of multi-source reliability information is not only related but also different, and make full use of the field test information, mixed Beta prior distribution with inheritance factor is introduced.

$$
\pi_{\rho}(R)=\sum_{i=1}^{n} \rho_{i} \pi_{i}(R),, \sum_{i=1}^{n} \rho_{i}=1
$$

In the formula, $\rho_{i}$ is the inheritance factor, and $0 \leq \rho_{i} \leq 1$.

\section{Determining the Inheritance Factor by the Goodness of Fit}

Inheritance factor can be considered as a measure of the similarity between the reliability information and the system test samples. There are two main methods to determine the inheritance factor. One is determined by the experience knowledge of experts [3]. This method is too subjective. Another method is to solve the problem according to the hierarchical Bayes method [4]. The problem faced by this method is its probability distribution and value range also need expertise to determine. Another reason to limit its application is this method needs to solve complex mathematical integral.

In order to solve the difficulties mentioned above, $a_{i}$ and $b_{i}$ can be seen as the prior test information due to the fact that the parameter $a_{i}$ and $b_{i}$ in each of the prior distributions are determined by their respective information sources. It can be considered that the inheritance factor 
$\rho_{i}$ is determined by the goodness of fit of the test information and the system field test information $\chi^{2}$.

The assumption of the i-th reliability information and system field test information are

$\left(m_{i}, y_{i}\right) \in Y,(n, x) \in X$

In the formula, $m_{i}, n$ is the number of tests, $x, y_{i}$ is the number of successful tests, $f_{i}^{\prime}=m_{i}-y_{i}$ is the number of failure tests, $f=n-x$ is the number of failure tests.

Null hypothesis $H_{0}: X$ and $Y$ come from the same population. The test statistic is

$$
K_{i}=\frac{\left(x f_{i}^{\prime}-y_{i} f\right)^{2}\left(m_{i}+n\right)}{\left(x+y_{i}\right)\left(f+f_{i}^{\prime}\right) m_{i} n}
$$

It can converge in distribution to a $\chi^{2}$ distribution whose degree of freedom is 1 . Under certain significant level, if

$$
\begin{aligned}
& K_{i}>\chi_{1}^{2}(\alpha) \Rightarrow \text { Reject } H_{0} \\
& K_{i} \leq \chi_{1}^{2}(\alpha) \Rightarrow \text { Accept } H_{0}
\end{aligned}
$$

In the formula, $\chi_{1}^{2}(\alpha)$ is the $\alpha$ fractile whose degree of freedom is 1 .

It should be noted here that the $x, y_{i}, f_{i}^{\prime}, f$ in the statistic $K_{i}$ should be more than 5 . It is generally difficult to achieve under the premise of small samples. Thus, a modified statistic $K_{i}^{\prime}$ is given in the literature [5].

$$
K_{i}{ }^{\prime}=\frac{\left[\left|x f_{i}^{\prime}-y_{i} f\right|-\frac{m_{i}-n}{2}\right]^{2}\left(m_{i}+n\right)}{\left(x+y_{i}\right)\left(f+f_{i}{ }^{\prime}\right) m_{i} n}
$$

If the null hypothesis is accepted, it cannot be explained that the null hypothesis must be true. In particular, it is pointed out that, under the condition of small samples, it is only able to explain that the evidence supporting the rejection of the null hypothesis is insufficient. Furthermore, the reason of the $K_{i}^{\prime}$ which is slightly smaller than $\chi_{1}^{2}(\alpha)$ for supporting $H_{0}$ is not so strong than the reason of the $K_{i}^{\prime}$ which is far smaller than $\chi_{1}^{2}(\alpha)$ for supporting $H_{0}$. Order the goodness of fit is

$$
Q\left(K_{i}{ }^{\prime}\right)=P\left(\chi_{1}^{2}(\alpha)>K_{i}{ }^{\prime}\right)
$$

$Q\left(K_{i}^{\prime}\right)$ represents a measure of the similarity between two samples. The larger the $Q\left(K_{i}^{\prime}\right)$ is, the bigger the similarity between prior reliability information and system field test information is. At the same time, it means the inheritance factor is much larger. According to the physical meaning of the inheritance factor, the inheritance factor can be expressed as

$$
\rho_{i}=\frac{Q\left(K_{i}{ }^{\prime}\right)}{Q\left(K_{1}{ }^{\prime}\right)+Q\left(K_{2}{ }^{\prime}\right)+\cdots+Q\left(K_{n}{ }^{\prime}\right)}
$$

\section{Statistical Analysis of Bayes Based on the Mixed Beta Distribution}

After obtaining field test information through system test $D \sim(N, S, F)$ ( $N$ is the number of test, $S$ is the number of successful tests, $F$ is the number of failure tests.), and determining the inheritance factor $\rho_{i}$, the posterior distribution can be derived

$$
\pi_{\rho}(R \mid D)=\frac{\sum_{i=1}^{n} \rho_{i} \frac{R^{S+a_{i}-1}(1-R)^{F+b_{i}-1}}{\beta\left(a_{i}, b_{i}\right)}}{\sum_{i=1}^{n} \rho_{i} \frac{\beta\left(s+a_{i}, f+b_{i}\right)}{\beta\left(a_{i}, b_{i}\right)}}
$$


For a given confidence level $\gamma$, the lower confidence limit can be obtained by the following formula.

$$
\int_{0}^{R_{L}} \pi_{\rho}(R \mid D)=1-\gamma
$$

\section{The Process of Reliability Assessment}

Reliability assessment of the success or failure products based on the multi-source information fusion technology with improved inheritance factor can be carried out according to the following steps.

(1) Collecting expertise information, components and subsystem information, reliability growth test information and field test information.

(2) Converting reliability information by using the information entropy method.

(3) Establishing the prior distribution and calculating the hyper-parameters, the test statistic $\mathrm{K}$ and the goodness of fit $\mathrm{Q}(\mathrm{K})$.

(4) Calculating the inheritance factor and establishing the posterior distribution

(5) Calculating the lower confidence limit under the given confidence level

\section{Example Analysis}

According to the literature [6] in evaluating the reliability of a certain type of solid rocket motor, based on the expertise information, components and subsystem information and reliability growth test information, combined with the field test information, the reliability information fusion calculation is carried out.

\section{Reliability Information}

\section{Expertise Information}

The reliability of solid rocket motor system was predicted by six experts. The trust degree of each expert is $0.80,0.75,0.90,0.75,0.95,0.80$. The reliability level of the system is divided into 5 levels. Using SR to show the reliability level, specific data is shown in table 1.

Table 1 . System reliability rating scale.

\begin{tabular}{|c|c|c|c|c|c|}
\hline SR & SR1 & SR2 & SR3 & SR4 & SR5 \\
\hline Reliability interval & {$[0.75,0.8]$} & {$[0.8,0.85]$} & {$[0.85,0.9]$} & {$[0.9,0.95]$} & {$[0.95,1]$} \\
\hline
\end{tabular}

The probability distribution function given by the 6 experts is

$$
\begin{aligned}
& m_{1}=(0.02,0.10,0.10,0.75,0.03) \\
& m_{2}=(0.00,0.05,0.02,0.70,0.05) \\
& m_{3}=(0.01,0.09,0.15,0.70,0.05) \\
& m_{4}=(0.02,0.08,0.10,0.75,0.05) \\
& m_{5}=(0.05,0.05,0.05,0.80,0.05) \\
& m_{6}=(0.00,0.10,0.20,0.70,0.00)
\end{aligned}
$$

\section{Components and Subsystem Information}

The simplified reliability block diagram of a certain type of solid rocket motor is shown in figure 1 . According to the corresponding reliability test plan, each component can obtain the success or failure type test data, as shown in table 2 . 


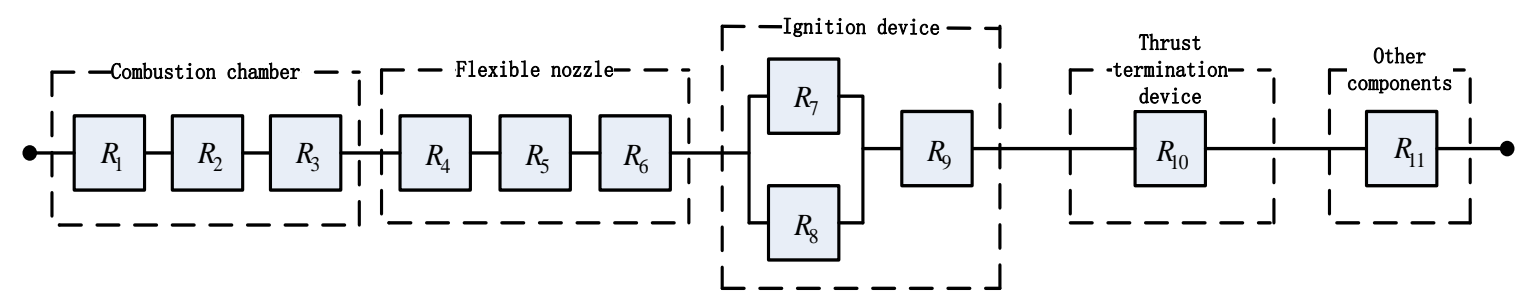

Fig. 1. The simplified reliability block diagram of a certain type of solid rocket motor.

Table 2. Component and subsystem test data.

\begin{tabular}{|c|c|c|c|c|}
\hline Components & $\begin{array}{c}\text { Name of } \\
\text { components and } \\
\text { subsystem }\end{array}$ & $\begin{array}{c}\text { The } \\
\text { number of } \\
\text { tests } n_{i}\end{array}$ & $\begin{array}{c}\text { The number of } \\
\text { successful tests } \\
s_{i}\end{array}$ & $\begin{array}{c}\text { The number } \\
\text { of failure tests } \\
f_{i}\end{array}$ \\
\hline$R_{1}$ & Grain & 165 & 164 & 1 \\
\hline$R_{2}$ & Shell & 20 & 20 & 0 \\
\hline$R_{3}$ & Other Components 1 & 98 & 98 & 0 \\
\hline$R_{4}$ & Throat & 85 & 84 & 1 \\
\hline$R_{5}$ & Nozzle & 90 & 89 & 1 \\
\hline$R_{6}$ & Other Components 2 & 80 & 79 & 1 \\
\hline$R_{7}$ & Tube for firing 1 & 100 & 99 & 1 \\
\hline$R_{8}$ & Tube for firing 2 & 100 & 99 & 1 \\
\hline$R_{9}$ & Other Components 3 & 74 & 74 & 0 \\
\hline$R_{10}$ & Thrust termination & 95 & 94 & 72 \\
\hline$R_{11}$ & Other Components 4 & 72 & & \\
\hline
\end{tabular}

\section{Reliability Growth Test Information}

The whole system reliability growth test of this type of solid rocket motor is carried out in 5 stages in development period. Test data at each stage are shown in table 3 and 4.

Table 3. Reliability growth data.

\begin{tabular}{|c|c|c|}
\hline Stage & The number of tests & The number of failure tests \\
\hline 1 & 6 & 3 \\
\hline 2 & 5 & 2 \\
\hline 3 & 4 & 1 \\
\hline 4 & 4 & 1 \\
\hline 5 & 5 & 0 \\
\hline
\end{tabular}

Table 4. Failure times and cumulative test times.

\begin{tabular}{|c|c|}
\hline Failure times & Cumulative test times \\
\hline 1 & 2 \\
\hline 2 & 5 \\
\hline 3 & 6 \\
\hline 4 & 9 \\
\hline 5 & 11 \\
\hline 6 & 15 \\
\hline 7 & 17 \\
\hline
\end{tabular}




\section{Field Test Information}

The whole system field test was 6 times, they are all successful. That is $(N, S, F)=(6,6,0)$.

\section{The Fusion of Multi-source Reliability Information}

\section{The Fusion of Expertise Information}

According to the searching optimization algorithm mentioned in literature [7], it can be obtained a prior distribution based on expertise information. Beta $\left(\mathrm{a}_{1}, \mathrm{~b}_{1}\right)$ is $\operatorname{Beta}(236.3,6.163)$. The hyperparameters of prior distribution is $\mathrm{a}_{1}=\mathrm{s}_{1}=236.3, \mathrm{~b}_{1}=\mathrm{f}_{1}=6.163$. Field test data is $(\mathrm{N}, \mathrm{S})=(6,6)$. After the calculation, the test statistic is $\mathrm{K}_{1}=0.8320$, the goodness of fit is $\mathrm{Q}\left(\mathrm{K}_{1}\right)=0.3617$.

\section{The Fusion of Components and Subsystem Information}

This type of solid rocket motor is composed of four main parts. In view of the above components and subsystem information, the classic pyramid assessment method and the information entropy method are used respectively. The results of information conversion are shown in table 5.

Table 5. The converted information of components and subsystem.

\begin{tabular}{|c|l|c|c|c|}
\hline & LM method & SR method & CMSR method & $\begin{array}{c}\text { Information } \\
\text { entropy method }\end{array}$ \\
\hline $\begin{array}{c}\text { The converted } \\
\text { information }\end{array}$ & $(20,1.022)$ & $(20.9471,0.9471)$ & $(53.7439,2.7387)$ & $(238.7685,12.2011)$ \\
\hline $\begin{array}{c}\text { Lower } \\
\text { confidence limit }\end{array}$ & 0.8176 & 0.8301 & 0.8861 & 0.9255 \\
\hline
\end{tabular}

Through the analysis of the assessment results, we can know that the assessment result based on SR method is conservative due to the information compression. The assessment result based on LM method is more conservative because the information compression of LM method is more serious than SR method. Although the CMSR method only completed information compression for one time, but there still has information loss, the assessment results are still conservative. The assessment method based on information entropy method does not exist information compression, so it is a better method to evaluate the reliability of the system by using the components and subsystem test information.

After the conversion, the equivalent system test information is (n, $\mathrm{s}$, $\mathrm{f})=(238.7685,226.5674,12.2011)$. The hyper-parameters of prior distribution is $\mathrm{a}_{2}=\mathrm{s} 2=226.5674$, $b_{2}=f_{2}=12.2011$. Field test data is $(N, S)=(6,6)$. After the calculation, the test statistic is $K_{2}=0.1456$, the goodness of fit is $\mathrm{Q}\left(\mathrm{K}_{2}\right)=0.7028$.

\section{The Fusion of Reliability Growth Test Information}

(1) The test of reliability growth trend

For each stage of reliability growth test information, the test of the growth trend is need to be done. The tests are censored with a successful test. So $J=24, M=7$ ( $J$ is the cumulative test number at the time of completion of the test. $M=f$ when the tests are censored with a successful test.) The Laplace statistic is $\mu=-1.036$. When the significant level is 0.2 ( $\alpha=0.2$ ), $\mu=-1.036 \leq-\mu_{\alpha}=1.282$. It indicates the reliability has obvious growth trend.

(2) The parameter estimation and goodness of fit test

The parameters of the model are calculated by parameter estimation. $\hat{\lambda}=0.8786, \hat{\beta}=0.6435$ And the goodness of fit test is done subsequently. The test statistic is $C_{M}^{2}=0.0753$. When the significant is $0.2(\alpha=0.2), C_{M}^{2}=0.0753 \leq C_{M, \alpha}^{2}=0.123$. It is considered that the model fit is good; the AMSAA 
model can be used to fit it

The products have been carried out for 5 stages of reliability growth test, based on the test information to calculate the parameter estimation of the discrete model $\hat{\lambda}, \hat{\beta}$. It can be considered that the system field test is the sixth stage. The number of field tests is $n_{6}=6$. The predictive reliability of the sixth stage is $\hat{R}=0.8252$. According to the construction method based on maximum entropy prior distribution, the prior distribution of the sixth stage is $B e(R \mid 3.404,0.7211)$. The posterior distribution is $B e(R \mid 9.404,0.7211)$. The estimated reliability after the field test is 0.9288 according to the reliability growth information. The lower confidence limit is $R_{L}=0.8237$ when the confidence level is 0.9 .

After the calculation, the test statistic is $\mathrm{K}_{3}=0.0812$, the goodness of fit is $\mathrm{Q}\left(\mathrm{K}_{3}\right)=0.9188$.

\section{The Fusion of Multi-Source Reliability Information}

According to the formula (5), the inheritance factor of three kinds of reliability information can be got respectively.

$$
\rho_{1}=0.1824, \rho_{2}=0.3543, \rho_{3}=0.4633 \text {. }
$$

The lower confidence limit of the system reliability is $R_{L}$ when the confidence level is 0.9 according to the formula (6) and (7).

$$
R_{L}=0.8975
$$

The comparative analysis of different assessment methods is carried out in order to illustrate the advantages of this method, as shown in table 6. Method 1 is non-stochastic confidence low limit which only contains field test information. Method 2 is the lower confidence limit based on information entropy method which is determined by the converted information of components and subsystem. Method 3 is the lower confidence limit based on Bayes method which is without consideration of the varied population between components, subsystem information and system field test information.

Table 6. Comparison of different assessment methods.

\begin{tabular}{|l|c|c|c|c|c|c|c|}
\hline $\begin{array}{l}\text { Assessment } \\
\text { method }\end{array}$ & $\begin{array}{c}\text { Method } \\
1\end{array}$ & $\begin{array}{c}\text { Method } \\
2\end{array}$ & $\begin{array}{c}\text { Method } \\
3\end{array}$ & $\begin{array}{c}\text { LM } \\
\text { method }\end{array}$ & $\begin{array}{c}\text { SR } \\
\text { method }\end{array}$ & $\begin{array}{c}\text { CMSR } \\
\text { method }\end{array}$ & $\begin{array}{c}\text { Information } \\
\text { Fusion }\end{array}$ \\
\hline $\begin{array}{l}\text { Lower } \\
\text { confidence limit }\end{array}$ & 0.6301 & 0.9255 & 0.9349 & 0.8176 & 0.8301 & 0.8861 & 0.8975 \\
\hline
\end{tabular}

From the assessment results we can draw the following conclusions. Method 1 only takes into account the system field test information, the assessment result is the most conservative. It even can't reach the threshold in the design period. It has little significance for the reliability assessment of small samples system. Method 2 does not contain the system field test information. Reliability assessment is carried out only based on the test information of components and subsystem. From the point of view of "system theory", it is clear that it is difficult to ensure accurate. This method has a certain risk that the assessment results are not agree with the fact. Method 3 is the Bayes method which uses the prior information and field test information to evaluate the system reliability. It does not take into account the problem that the prior information and field test information may have varied population problem. It also has a certain risk that the assessment results are not agree with the fact. LM method and SR method are both classical reliability assessment methods, the assessment result is conservative because of the information compression. Although the CMSR method has a better theory, it still has the information compression in the assessment process, which leads to a still conservative assessment result. The multi-source information fusion method based on improved inheritance factor not only take into account the inheritance between prior information and field test information but also takes 
into account their differences. The assessment result is more reasonable.

\section{Conclusion}

By aiming at the reliability assessment characteristic of complex system with small samples, this paper presents the reliability assessment method based on multi-source information fusion technology with improved inheritance factor. It not only takes into account the inheritance of prior reliability information and system field test information, but also distinguishes the varied population problem between these two. The rationality of assessment results is verified through comparison among different assessment methods. Moreover, goodness-of-fit test method is used to determine the inheritance factor, which can reduce the influence of human factors. Therefore, it has very high application values in the field of reliability engineering.

\section{References}

1. W. Huawei, G. Jun, Complex systems reliability analysis and assessment (Science Press, Beijing, 2013)

2. S. Youchao, J. Appl. Sci., 19, 113 (2001)

3. A. Kleyner, S. Bhagath, M. Gasparini, Microelectron. Reliab., 37, 879 (1997)

4. Z. Shifeng, F. Shujiang, Z. Jinhuai, Acta Arma., 22, 238 (2001)

5. R.A. Fisher, F. Yates, Statistical tables for biological agricultural and medical research (Oliver\&Boyd, Edingburgh, 1957)

6. L. Jing, Solid Rocket Motor Reliability Evaluation Based on BAYES Method (NUDT, ChangSha, 2008)

7. M. Zhibo, Z. Jianxin, X. Naixin, Chin. J. Comput. Phys., 20, 391 (2003)

8. C. Hao, Z. Yang, G. Bo, J. Ping, Mech. Sci. Technol. Aerosp. Eng., 35, 11392016

9. W. Wei, Z. Haiyun, Y. Guojun, Syst. Eng. Theor. Pract., 9, 142 (2005)

10. L. Han, Research on Methods of Reliability Assessment with Small Sample Test on Basis of Bayes Theory (NUDT, ChangSha, 2006)

11. Y. Huiyun, J. Xi'an Univ. Arts Sci., 11, 422008

12. Y. Jun, H. Jin, S. Lijuan, Z. Yu, J. Beijing Univ. Aeron. Astron., 35, 786, 2009 